\title{
Gefährliche Flüchtlinge?! Wie das europäische Asyl- recht mit mutmasslichen Terroristen umgeht
}

Andrea Egbuna-Joss *

Asylsuchende und Flüchtlinge werden von westlichen Staaten vermehrt als sicherheitspolitische Bedrohung wahrgenommen. Seit den Terroranschlägen vom 11. September 2001 in den USA hat sich diese Tendenz kontinuierlich verstärkt, und die öffentliche Diskussion über die Aufnahme von Schutzsuchenden ist heute oft von der Befürchtung geprägt, dass Flüchtlinge und weitere schutzsuchende Personen durch ihre Anwesenheit im Aufnahmestaat dessen öffentliche Sicherheit und Ordnung gefährden. Angesichts der zunehmenden Anzahl terroristischer Anschläge werden nicht nur verstärkte polizeiliche Massnahmen, sondern oftmals auch eine Verschärfung der Asylgesetzgebung propagiert. Die kürzlich erschienene Dissertation der Autorin untersucht, wie die Europäische Union im Rahmen der Harmonisierung des Europäischen Asylrechts auf mutmasslich gefährlichen Asylsuchende reagiert hat, und ob die relevanten Bestimmungen mit den völkerrechtlichen Vorgaben im Einklang stehen.

I. Einleitung 103

II. Der Schutz der öffentlichen Sicherheit im gemeinsamen Europäischen

Asylsystem 104

III. Der Schutz der öffentlichen Sicherheit im Rahmen der QualifikationsRichtlinie 2011/95 106

1. Der Ausschluss mutmasslich gefährlicher Personen von der internationalen Schutzgewährung 106

2. Die Einschränkung der Statusrechte zum Schutz der öffentlichen Sicherheit. 108

3. Die Beendigung der Schutzgewährung aus Sicherheitsüberlegungen .......... 109

IV. Würdigung 111

Zitiervorschlag: Andrea Egbuna-Joss, Gefährliche Flüchtlinge?! Wie das europäische Asylrecht mit mutmasslichen Terroristen umgeht, in: sui-generis 2017, S. 102

URL:

sui-generis.ch $/ 38$

DOI:

https://doi.org/10.21257/sg.38

* Dr. iur. Andrea Egbuna-Joss, wissenschaftliche Mitarbeiterin, Schweizerisches Kompetenzzentrum für Menschenrechte sowie Lehrstuhl für Völkerrecht, Universität Freiburg i.Ue.

Dieses Werk ist lizenziert unter einer Creative Commons Namensnennung - Weitergabe unter gleichen Bedingungen 4.0 International Lizenz. 


\section{Einleitung1}

1 Flüchtlinge und Schutzsuchende sind Personen, die Opfer von Verfolgung, Krieg und schwersten Menschenrechtsverletzungen geworden oder davon bedroht sind. Ist ihr Herkunftsstaat nicht in der Lage oder nicht willens, diese Personen zu schützen, räumt ihnen das Völkerrecht unter gewissen Bedingungen einen Anspruch auf internationale Schutzgewährung in einem anderen Staat ein.

Seit den Terroranschlägen vom 11. September 2001 in den USA sind die Medienberichterstattung und politische Diskussion über Flüchtlinge in westlichen Staaten aber oftmals nicht mehr von deren Schutzbedürfnis, sondern von dem von ihnen angeblich ausgehenden $\mathrm{Si}$ cherheitsrisiko geprägt. In seiner Reaktion auf die Terroranschläge vom 11. September 2001 in den USA rief der UNSicherheitsrat mit der bindenden Resolution 1373 (2001) die Staaten nicht nur dazu auf, eine Reihe von Massnahmen zur Bekämpfung des internationalen Terrorismus zu ergreifen, sondern gleichzeitig auch dazu, die Ausschlussklauseln der Genfer Flüchtlingskonvention (GFK²) strikt anzuwenden und sicherzustellen, dass die Asylgewährung nicht von Personen missbraucht werde, um terroristische Taten zu begehen, zu organisieren oder zu erleichtern. Obwohl keiner der Attentäter sich vor den Anschlägen als Flüchtling oder Asylsuchender in den USA aufgehalten hatte, scheinen Schutz-

Die folgenden Ausführungen sind in leicht überarbeiteter Form übernommen aus Andrea Egbuna-Joss, Der Schutz der öffentlichen Ordnung und Sicherheit im Rahmen der internationalen Schutzgewährung, Diss. Freiburg i.Ue., Berlin/ Bern 2016 (insbesondere $\S \S 1,15$ und 16).

2 Abkommen vom 28. Juli 1951 über die Rechtsstellung der Flüchtlinge (GFK, SR 0.142.30). suchende seither immer wieder mit dem internationalen Terrorismus in Verbindung gebracht zu werden, und zwar nicht als dessen Opfer, sondern vielmehr als Verursacher und Täter. Als bedauerliche Konsequenz ist mittlerweile der Eindruck entstanden, dass alle Schutzsuchende potentiell gefährlich sind3. Das jüngste Beispiel dafür ist der von US-Präsident Trump Ende Januar 2017 verfügte Aufnahmestopp für syrische Flüchtlinge. So solle unter anderem verhindert werden, dass mit den Flüchtlingen auch radikalislamistische Terroristen in die USA einreisen und dort terroristische Anschläge verüben könnten4.

3 Aber wie geht das Asylrecht tatsächlich mit mutmasslich gefährlichen Flüchtlingen um? Haben diese überhaupt Anspruch auf Asylgewährung? Und sind im Rahmen des «Kampfes gegen den Terrorismus» weitere Verschärfungen der Asylgesetzgebung oder gar vorübergehende Einreisesperren gerechtfertigt?

4 Sicherheitsüberlegungen haben im Flüchtlings- und Asylrecht nicht erst seit Beginn dieses Jahrtausends und insbesondere nicht erst seit den Terroranschlägen vom 11. September 2001 eine Rolle gespielt. Die Staaten waren sich der Gefahr, dass nicht nur «echte» Flüchtlinge, sondern auch sogenannte «Justizflüchtlinge» und asylunwürdige Personen wie z.B. Kriegsverbrecher oder Mitglieder terroristischer Organisationen um Schutz ersuchen könnten, seit jeher

3 Vgl. Penelope Mathew, Resolution 1373 - A Call to Preempt Asylum Seekers? (or «Osama, the Asylum Seeker»), in: Jane McAdam, Forced Migration, Human Rights and Security, 2006, S. 1961, S. 33.

4 Executive Order: Protecting the Nation from Foreign Terrorist Entry into the United States, 27. Januar 2017, Section 5. 
bewusst. Die Gefahr des Missbrauchs des internationalen Schutzsystems durch solche Personen wurde damals jedoch nicht als Grund interpretiert, die Schutzwürdigkeit aller Flüchtlinge in Zweifel zu ziehen. Vielmehr sahen sich die Staaten veranlasst, in fast alle internationalen und regionalen Abkommen entsprechende Bestimmungen aufzunehmen, um solche Personen von der Schutzgewährung auszuschliessen oder sie unter eng festgelegten Bedingungen und bei besonderer Gefährlichkeit auch trotz Schutzbedürftigkeit wieder aus ihrem Staatsgebiet auszuweisen, d.h. vom völkerrechtlich garantierten Refoulement-Schutz auszunehmen. Die Genfer Flüchtlingskonvention von 1951 erwies sich dabei als dasjenige völkerrechtliche Abkommen, welches die differenziertesten Mechanismen zum Schutz der Sicherheit der Aufnahmestaaten und der internationalen Gemeinschaft vorsieht5.

Nichtsdestotrotz wird aktuell vermehrt die Frage aufgeworfen, ob internationale Instrumente wie die Genfer Flüchtlingskonvention und die völkerrechtlichen Abkommen zum Schutz der Menschenrechte auch den heutigen sicherheitspolitischen Herausforderungen nach wie vor angemessen Rechnung tragen können ${ }^{6}$. Immer häufiger berufen sich Aufnahmestaaten auf ihre Souveränität und ihre Schutzverpflichtungen gegenüber den ei-

\footnotetext{
Egbuna-Joss (Fn. 1), S. 68 ff.

Vgl. Brian Gorlick, Brian, (Mis)perception of Refugees, State Sovereignty and the Continuing Challenge of International Protection, in: Bayefsky, Anne F. (ed.), Human Rights and Refugees, Internally Displaced Persons and Migrant Workers, Essays in Memory of Joan Fitzpatrick and Arthur Helton, 2006, S. 65-89, S. 67; Alice Edwards, Human Rights, Refugees, and the Right «To Enjoy» Asylum, IJRL (2005), S. 293-330, S. $293 \mathrm{ff}$.
}

genen Staatsangehörigen, um Einschränkungen der völker- und menschenrechtlichen Garantien gegenüber ausländischen Staatsangehörigen im Allgemeinen und Asylsuchenden und Flüchtlingen im Besonderen zu rechtfertigen?.

\section{Der Schutz der öffentlichen Sicher- heit im gemeinsamen Europäi- schen Asylsystem}

6 Sicherheitsüberlegungen haben von Anfang an auch das gemeinsame Vorgehen der EU-Mitgliedstaaten im Bereich der Asylpolitik geprägt. Die ersten gemeinsamen asylrechtlichen Regelungen zu Beginn der Neunziger Jahre sollten primär das Sicherheitsdefizit kompensieren, welches durch den Wegfall der Binnengrenzkontrollen entstehen würde ${ }^{8}$. Sekundärbewegungen von Flüchtlingen und Schutzsuchenden innerhalb der Europäischen Union wurden in erster Linie als ein Problem der illegalen Einreise und Weiterreise von Drittstaatsangehörigen betrachtet, welches mit den ausgearbeiteten Zuständigkeitsregeln zur Behandlung eines Asylgesuchs bekämpft werden sollten 9 .
$7 \longdiv { \text { So kündigte etwa die britische Premierministerin } }$ Theresa May anfangs Juni 2017 an, sie wolle es den Behörden erleichtern «to deport foreign terrorist suspects back to their own countries» und «to restrict the freedom and movements of terrorist suspects when we have enough evidence to know they are a threat, but not enough evidence to prosecute them in full in court. And if our human rights laws get in the way of doing it, we will change the law so we can do it.» bbc.com, Theresa May: Human rights laws could change for terror fight, 7. Juni 2017.

8 Egbuna-Joss (Fn. 1), S. 75 f.

9 Die Zuständigkeitsregeln waren im Schengener Durchführungsübereinkommen sowie dem Dubliner Übereinkommen von 1990 enthalten; S. dazu Egbuna-Joss (Fn. 1), S. 76 ff. 
7 Nach Inkrafttreten des Amsterdamer Vertrages ${ }^{10}$ am 1. Mai 1999 ist es in der Folge auch im Rahmen der Asylrechtsharmonisierung $\mathrm{zu}$ einer Vermischung von sicherheitspolitischen und humanitären Anliegen gekommen. Bis heute scheint das gemeinsame Vorgehen der EU-Mitgliedstaaten im Asylbereich stark von den übergeordneten Zielen eines effektiven Aussengrenzschutzes und der Bekämpfung der irregulären Einreise und Weiterreise von Flüchtlingen und Schutzsuchenden, und weit weniger von humanitären Motiven geprägt, auch wenn die Europäische Union zumindest offiziell stets den dem europäischen Asylrecht zu Grunde liegenden Schutzgedanken und die Wichtigkeit der Einhaltung der völkerrechtlichen Vorgaben betont.

Diese Vorgaben waren auch bei der Ausarbeitung der sogenannten Qualifikationsrichtlinie über die Anerkennung von Personen als Flüchtlinge oder subsidiär Schutzberechtigte (im Folgenden: RL 2011/95) ${ }^{11}$ zu beachten. Neben der Asylgewährung für Flüchtlinge sollen grundsätzlich diejenigen Personen sogenannt subsidiären Schutz erhalten, welche nicht Flüchtlinge im rechtlichen Sinn sind, bei einer Rückkehr in ihr Heimatland jedoch

10 Vertrag von Amsterdam zur Änderung des Vertrages über die Europäische Union, der Verträge zur Gründung der Europäischen Gemeinschaften sowie einiger damit zusammenhängender Rechtsakte, ABl. C 340/1997, S. 1-144; Vertrag zur Gründung der Europäischen Gemeinschaft (Amsterdam konsolidierte Fassung) ABl. C 340/1997, S. 173-306.

11 Richtlinie 2011/95 über Normen für die Anerkennung von Drittstaatsangehörigen oder Staatenlosen als Personen mit Anspruch auf internationalen Schutz, für einen einheitlichen Status für Flüchtlinge oder für Personen mit Anrecht auf subsidiären Schutz und für den Inhalt des zu gewährenden Schutzes (Neufassung), ABl. 337/ 2011, S. 9. schwere Menschenrechtsverletzungen befürchten müssen (Art. 2 lit.f $\mathrm{RL}$ 2011/95) ${ }^{12}$. Die Qualifikationsrichtlinie enthält allgemeine Vorgaben für die Prüfung von Anträgen auf internationalen Schutz (Art. 4-8 RL 2011/95), legt die Bedingungen für die Anerkennung als Flüchtlinge (Art. 9-14 RL 2011/95) und die Gewährung subsidiären Schutzes (Art. 15-19 RL 2011/95) fest und regelt den Inhalt des internationalen Schutzes (Art. 20-35 RL 2011/95). Während die erste Fassung von 2004 lediglich Mindestnormen festlegte, soll die revidierte Richtlinie von 2011 nun einheitliche Vorgaben zur Prüfung der Anträge und einen einheitlichen Status festlegen. Viele Bestimmungen der ersten Fassung wurden jedoch unverändert in die RL 2011/95 übernommen ${ }^{13}$. So auch die zahlreichen Bestimmungen, welche vor dem Hintergrund der verstärkten Massnahmen zur Bekämpfung des internationalen Terrorismus in die erste Fassung von 2004 aufgenommen wurden. Diese Bestimmungen zielen direkt oder indirekt auf den Schutz der öffentlichen Sicherheit und Ordnung und sehen zu diesem Zweck Einschränkungen der Rechte von Flüchtlingen und Schutzsuchenden vor. Im Folgenden sollen diese Bestimmungen dargestellt und kurz auf ihre Kompatibilität mit den völkerrechtlichen Vorgaben untersucht werden.

$12 \overline{\mathrm{Zu} \text { den unterschiedlichen Konzepten der subsidi- }}$ är Schutzberechtigten im europäischen Recht und den vorläufig aufgenommenen Personen in der Schweiz s. etwa Patricia Petermann/ Christine Kauffmann, Die subsidiäre Schutzform, in: UNHCR/ Schweizerische Flüchtlingshilfe (Hrsg.), Schweizer Asylrecht, EU-Standards und internationales Flüchtlingsrecht, Eine Vergleichsstudie, 2009, 67-122.

13 Egbuna-Joss (Fn. 1), S. 143 m.w.H. 
III. Der Schutz der öffentlichen Sicherheit im Rahmen der Qualifikationsrichtlinie 2011/95

9 Die Bestimmungen der Qualifikationsrichtlinie 2011/95, welche dem Schutz der öffentlichen Sicherheit und Ordnung dienen oder sich (indirekt) darauf auswirken können, lassen sich in drei Kategorien einteilen:

1. Bestimmungen über den Ausschluss gewisser Personen von der Anerkennung als Flüchtlinge oder von der Gewährung subsidiären Schutzes,

2. Bestimmungen, welche die Einschränkung der Statusrechte aus Gründen der öffentlichen Sicherheit und Ordnung vorsehen, sowie

3. Bestimmungen über die Beendigung der internationalen Schutzgewährung aus Gründen der öffentlichen Sicherheit und Ordnung.

\section{Der Ausschluss mutmasslich ge-} fährlicher Personen von der internationalen Schutzgewährung

Nicht alle Personen, die internationalen Schutzes bedürfen, sollen auch als Flüchtlinge anerkannt werden: Personen, die in ihrem Herkunftsstaat zwar von Verfolgung oder von schweren Menschenrechtsverletzungen bedroht sind, jedoch vor ihrer Gesuchstellung mutmasslich schwere Verbrechen begangen haben, gelten gemäss den Bestimmungen der Genfer Flüchtlingskonvention (Art. 1 F GFK) und auch der Richtlinie 2011/95 (Art. 12 Abs. 2 lit. a-c) als schutzunwürdig und sollen daher von der Anerkennung als Flüchtlinge ausgeschlossen werden. Durch den Ausschluss dieser Personen soll der Missbrauch der durch die Qualifikationsrichtlinie gewährten Rechte verhindert und sichergestellt wer- den, dass diese Personen nicht auf dem «Asylweg» einer strafrechtlichen Verfolgung entgehen können ${ }^{14}$.

11 Im Zusammenhang mit der Terrorismusbekämpfung scheint in den letzten Jahren insbesondere der Ausschluss aufgrund von Handlungen, die den Zielen und Grundsätzen der UN zuwiderlaufen (Art. $1 \mathrm{~F}$ lit. c GFK bzw. Art. 12 Abs. 2 lit. c RL 2011/95), an Attraktivität gewonnen zu haben. Dazu lässt sich zusammenfassend festhalten, dass dieser Ausschlussgrund im Hinblick auf die Normadressaten und die erfassten Taten weiterhin von vielen begrifflichen Unsicherheiten geprägt ist. Grundsätzlich sollte er im Verhältnis zu den übrigen Ausschlussgründen nur subsidiär zur Anwendung kommen. Mutmassliche Terroristen können zwar von diesem Ausschlussgrund erfasst werden, allerdings bleibt die sorgfältige Prüfung der Umstände des Einzelfalles weiterhin unabdingbar. Nicht zulässig ist daher gemäss EuGH ein automatisierter Ausschluss von Personen, die z.B. auf sogenannten Terrorlisten des UN-Sicherheitsrates oder der EU aufgeführt sind ${ }^{15}$.

12 Die Ausschlussgründe in Art. 12 Abs. 2 lit. a-c RL 2011/95 finden auch auf Personen Anwendung, die sich an den genannten Taten beteiligt haben (Art. 12 Abs. 3 RL 2011/95). Dieses Vorgehen scheint insbesondere bei den schwersten internationalen Verbrechen und ähnlich schwerwiegenden Handlungen gerechtfertigt. Erforderlich bleibt allerdings, dass die Beteiligungshandlung eine ge-

14 Egbuna-Joss (Fn. 1), S. 160 m.w.H.

15 Urteil des EuGH C-57/o9 und C-101/09 vom 9. November 2010 (B. und D. gegen Deutschland), Rn. 91-99; vgl. Egbuna-Joss (Fn. 1), S. 209 f., S. $211 \mathrm{ff}$. 
wisse Erheblichkeit für die Begehung der Haupttat aufweist. Und so werden auch im Zusammenhang mit der Terrorismusbekämpfung bloss unerhebliche Unterstützungshandlungen von Sympathisanten einer terroristischen Organisation nicht von den Ausschlussklauseln erfasst. Im Gegenzug ist es aber auch nicht erforderlich, dass der Antragsteller persönlich und durch eigene Gewaltbeiträge terroristische Verbrechen begangen haben muss. Die Anforderungen an einen Nachweis der persönlichen Beteiligung sinken zudem, je gewalttätiger die terroristische Organisation ist: Sofern ein Gesuchsteller freiwillig Mitglied einer besonders gewalttätigen terroristischen Organisation ist, wird generell vermutet, dass er in irgendeiner Weise einen entscheidenden Beitrag zur Ausführung gewalttätiger Handlungen geleistet hat. In derartigen Fällen kommt es zu einer Beweislastumkehr und der Antragsteller muss beweisen, dass er weder Kenntnis von den strafbaren Handlungen hatte noch einen wesentlichen Beitrag zu diesen geleistet hat ${ }^{16}$.

Die Qualifikationsrichtlinie 2011/95 überträgt die Ausschlussgründe der Flüchtlingskonvention in leicht modifizierter und zusätzlich erweiterter Form auch auf die subsidiäre Schutzgewährung (Art. 17 RL 2011/95). Solange dadurch das menschenrechtliche Minimum nämlich das absolute Verbot der $\mathrm{Zu}$ rückweisung, Abschiebung oder Auslieferung in einen Staat, in dem Folter oder andere unmenschliche oder erniedrigende Behandlung oder Bestrafung drohen nicht berührt wird, ist dieses Vorgehen aus völkerrechtlicher Sicht zwar bedenklich, grundsätzlich aber zulässig. Da die

16 Vgl. Egbuna-Joss (Fn. 1), S. 214 m.w.H.
Ausschlussklauseln des Art. 17 RL 2011/ 95 wesentlich weiter gefasst sind als diejenigen des Art. 12 Abs. 2 RL 2011/95, ist eine Person, die von der Anerkennung als Flüchtling ausgeschlossen ist, automatisch auch von der subsidiären Schutzgewährung ausgeschlossen. Folglich entfällt dadurch - zumindest bei Personen, die von einem oder mehreren Ausschlussgründen erfasst werden - die Funktion des «ergänzenden» menschenrechtlichen Schutzes, als welcher der subsidiäre Schutz ursprünglich konzipiert war.

14 Darüber hinaus formuliert die Qualifikationsrichtlinie auch die Ausnahme vom flüchtlingsrechtlichen Verbot der Rückweisung ins Herkunftsland um, wenn die Person eine Gefahr für die Allgemeinheit oder die Sicherheit des Aufnahmestaates darstellt (Art. 33 Abs. 2 GFK), und schafft im Rahmen der subsidiären Schutzgewährung einen zusätzlichen, zwingenden Ausschlussgrund. Eine Rückweisung gemäss Qualifikationsrichtlinie ist bereits dann gerechtfertigt, wenn «schwerwiegende Gründe die Annahme rechtfertigen", dass die schutzsuchende Person ein ernsthaftes Sicherheitsrisiko darstellt. Im Rahmen des internationalen Menschenrechtsschutzes ist eine solche Ausnahme aber gerade auch für mutmassliche Terroristen weder vorgesehen noch zulässig17. Hier wird deutlich, dass die Gefahr besteht, dass durch die Qualifikationsrichtlinie völkerrechtliche Konzepte vermischt werden.

15 Hinsichtlich der Beweisanforderungen im Rahmen der Ausschlussklauseln von

$17 \overline{\text { Zur relevanten Rechtsprechung des EGMR s. den }}$ Überblick bei Egbuna-Joss (Fn. 1), S. 55 ff. m.w.N. 
der Flüchtlingseigenschaft hat die Qualifikationsrichtlinie in Art. 12 Abs. 2 RL 2011/95 die Formulierung von Art. 1 F GFK («wenn schwerwiegende Gründe die Annahme rechtfertigen») übernommen, ohne diese zusätzlich zu präzisieren. Diese Beweisanforderungen wurden gleichzeitig auch für den Ausschluss von der subsidiären Schutzgewährung gemäss Art. 17 Abs. 1 RL 2011/95 für massgeblich erklärt. Die Flüchtlingskonvention selbst enthält keine Hinweise zur Auslegung des unbestimmten Rechtsbegriffes der «schwerwiegenden Gründe», und auch die bisherige Rechtsprechung des EuGH zu den Beweisanforderungen ist wenig aufschlussreich. Im Ergebnis gibt die unbestimmte Formulierung den rechtsanwendenden Behörden somit ein grosses Ermessen und überträgt ihnen die Kompetenz für quasi-judikative Entscheidungen, was gerade angesichts der schwerwiegenden Konsequenzen eines Ausschlusses als sehr problematisch erscheint ${ }^{18}$.

\section{Die Einschränkung der Statusrech- te zum Schutz der öffentlichen Si- cherheit}

Die Qualifikationsrichtlinie gewährt als erstes verbindliches internationales Instrument nicht nur anerkannten Flüchtlingen, sondern auch subsidiär Schutzberechtigten eine Reihe materieller Statusrechte, was als eine bedeutende Verbesserung des internationalen Schutzes zu bewerten ist. Denn während die Flüchtlingskonvention zumindest gewisse Grundsätze zur Rechtsstellung anerkannter Flüchtlinge im Aufnahmestaat enthält (wie den gleichberechtigten Zugang zum Arbeitsmarkt oder der öffentlichen Für- sorge), lassen sich aus den menschenrechtliche Verträgen lediglich ein Bleiberecht und der Grundsatz der menschenwürdigen Behandlung herleiten. Eigentliche Statusrechte für Personen, welchen in ihrem Herkunftsstaat schwere Menschenrechtsverletzungen drohen, gewährt das Völkerrecht jedoch nicht.

17 Die Qualifikationsrichtlinie 2011/95 wollte auch für diese Personen einen in allen Mitgliedstaaten einheitlichen Status gewährleisten. Gemäss Erwägungsgrund 12 besteht das wesentliche Ziel der Richtlinie darin «sicherzustellen, dass diesen [schutzbedürftigen] Personen in allen Mitgliedstaaten ein Mindestniveau von Leistungen geboten wird». Dieses Mindestniveau für Flüchtlinge und subsidiär Schutzbedürftige soll insbesondere auch den Zugang zu Leistungen der Sozialhilfe und der medizinischen Versorgung umfassen (Erwägungsgründe 45, $46 \mathrm{RL}$ 2011/95).

18 Aus Gründen der öffentlichen Sicherheit und Ordnung können einige dieser Statusrechte aber auch eingeschränkt oder gar verweigert werden: Aus Sicherheitsgründen kann z.B. die Erteilung eines Aufenthaltstitels verweigert und ein bereits erteilter Aufenthaltstitel widerrufen oder nicht verlängert werden ${ }^{19}$. Die Richtlinie sieht ähnliche Einschränkungen auch hinsichtlich der Erteilung von Reisedokumenten vor ${ }^{20}$. Diese Ausnahmetatbestände sind grundsätzlich restriktiv auszulegen. Wenn nur «zwingende» Gründe eine Einschränkung zulassen (wie in Art. 24, 25 RL 2011/95), sind die Anforderungen noch höher anzusetzen.

19 S. hierzu Egbuna-Joss (Fn. 1), S. 240 ff. m.w.H.

20 S. hierzu Egbuna-Joss (Fn. 1), S. 244 ff. m.w.H.

18 S. ausführlich Egbuna-Joss (Fn. 1), S. 226 ff. 
19 Eine sorgfältige Analyse kommt zum Ergebnis, dass die vorgesehenen Einschränkungen der Statusrechte von Flüchtlingen im Einklang mit den Vorgaben der Flüchtlingskonvention stehen ${ }^{21}$. Da das menschenrechtliche Rückweisungsverbot aus Sicherheitsgründen nicht einschränkbar ist, ist Flüchtlingen und subsidiär Schutzberechtigten, welche die Voraussetzungen gemäss der Rechtsprechung des EGMR zu Art. 3 EMRK 22 erfüllen, ein Bleiberecht im jeweiligen Mitgliedstaat auch dann zu gewähren, wenn ihnen gemäss RL 2011/95 aus Sicherheitsüberlegungen kein Aufenthaltstitel ausgestellt oder ein solcher entzogen wurde. Die Garantien der EU-Grundrechtscharta, welche insbesondere die Wahrung der Menschenwürde, die Achtung des Privat- und Familienlebens sowie die Rechte des Kindes verankert, sichern in solchen Fällen den schutzberechtigten Personen eine menschenwürdige Behandlung und ein gewisses Mindestmass an Schutz zu.

\section{Die Beendigung der Schutzgewäh- rung aus Sicherheitsüberlegungen}

Erweist sich ein Flüchtling oder eine subsidiär schutzberechtigte Person als Sicherheitsrisiko und kann diesem Risiko mit straf- und sicherheitsrechtlichen Massnahmen des nationalen Rechts (vgl. Art. 2 und 9 GFK) nicht angemessen begegnet werden, kann die internationale Schutzgewährung auch beendet werden. Die betroffene Person bleibt aus rechtlicher Sicht weiterhin Flüchtling, verliert aber ihr Aufenthaltsrecht im Aufnahmestaat.

21 S. ausführlich Egbuna-Joss (Fn. 1), S. 234 ff.

22 Konvention vom 28. November 1974 zum Schutze der Menschenrechte und Grundfreiheiten (EMRK; SR 0.101).
21 Bereits die Flüchtlingskonvention sieht in Art. 32 vor, dass aus Gründen der Staatssicherheit ein anerkannter Flüchtling aus dem Aufnahmestaat ausgewiesen werden kann. Vorausgesetzt wird, dass der Ausweisungsentscheid in einem gesetzlich vorgeschriebenen Verfahren getroffen wird und der Flüchtling Beweise zu seiner Entlastung vorbringen kann, sofern keine zwingenden Gründe der Staatssicherheit dem entgegenstehen (Art. 32 Abs. 2 GFK). Muss der Flüchtling den Aufnahmestaat verlassen, ist ihm eine angemessene Frist einzuräumen, um ihm zu ermöglichen, in ein anderes Land einzureisen. Während dieser Ausreise, sind zusätzliche (sichernde) Massnahmen, die für notwendig befunden werden (z.B. eine Ausschaffungshaft), ausdrücklich erlaubt (Art. 32 Abs. 3 GFK).

22 Die Ausweisung in den Verfolgerstaat ist grundsätzlich verboten (sog. Refoulement-Verbot, Art. 33 Abs. 1 GFK). Einzig wenn der Flüchtling als Gefahr für die Sicherheit des Aufnahmelandes oder als Bedrohung für die Gemeinschaft angesehen werden muss, weil er wegen eines besonders schweren Verbrechens rechtskräftig verurteilt wurde, ist es ihm verwehrt, sich auf das Refoulement-Verbot zu berufen (Art. 33 Abs. 2 GFK).

23 Die Qualifikationsrichtlinie folgt dieser klaren Systematik der Flüchtlingskonvention jedoch nicht und vermischt Ausschluss- und Ausweisungsgründe. Gemäss Art. 14 Abs. 4 RL 2011/95 kann die Flüchtlingseigenschaft aus Sicherheitsgründen aberkannt oder beendet werden. Anstatt auf Art. 32 GFK bezieht sich die Richtlinie in dieser Bestimmung aber auf die Ausnahmetatbestände des Refoule- 
ment-Verbotes in Art. 33 Abs. 2 GFK. Diese falsche Bezugnahme ist systematisch unsauber; aufgrund der höheren inhaltlichen Anforderungen von Art. 33 Abs. 2 GFK und unter Vorbehalt der Verfahrensgarantien von Art. 32 GFK bleibt sie aus völkerrechtlicher Sicht aber zulässig. Irreführend scheint hier einzig die Formulierung der «Aberkennung der Flüchtlingseigenschaft» zu sein, bleibt die Person aus völkerrechtlicher Sicht doch trotz Ausweisung Flüchtling.

24 Aus denselben Gründen (Gefahr für die Sicherheit oder eine Bedrohung der Gemeinschaft) und solange noch kein Anerkennungsentscheid getroffen wurde, können die Mitgliedstaaten im Weiteren davon absehen, einem Flüchtling den unionsrechtlichen Status als Flüchtling überhaupt zuzuerkennen (Art. 14 Abs. 5 RL 2011/95). Faktisch stellt diese Variante einen zusätzlichen Ausschlussgrund dar und ist nicht völkerrechtskonform. Dieselbe Schlussfolgerung muss auch für die nachträgliche Anwendung der Ausschlussgründe von Art. 12 (Art. 14 Abs. 3 lit. a RL 2011/95), d.h. die Sanktionierung von schutzunwürdigem Verhalten des Flüchtlings nach der Zuerkennung der Flüchtlingseigenschaft gelten.

Die Richtlinie stellt es den Mitgliedstaaten frei, von den Vorgaben der Flüchtlingskonvention abzuweichen, anstatt eindeutig völkerrechtskonforme Lösungen vorzusehen. Die Bedenken hinsichtlich der Völkerrechtskonformität der Beendigungsklauseln versucht sie durch sprachliche Anpassungen zu entschärfen. So wird die Umsetzung ins Ermessen der Mitgliedstaaten gestellt, welche selbstverständlich weiterhin vollumfänglich an ihre Verpflichtungen als Vertragsstaaten der GFK gebunden sind. Das Vorgehen des Unionsgesetzgebers, potentiell klar völkerrechtswidrige Varianten überhaupt vorzusehen, deren Umsetzung dann aber ins Ermessen der Staaten zu stellen, ist eher fragwürdig und als scheinbare Legalisierung eines nicht völkerrechtskonformen Verhaltens zu kritisieren ${ }^{23}$.

26 Im Rahmen der subsidiären Schutzgewährung sind Personen, welche eine Gefahr für die Sicherheit oder eine Bedrohung der Gemeinschaft darstellen, wie bereits erwähnt ${ }^{24}$ zwingend von der $\mathrm{Zu}$ erkennung des subsidiären Schutzstatus auszuschliessen (Art. 17 Abs. 1 lit. d). Die Ausschlussgründe in Art. 17 RL 2011/95 kommen zudem auch nachträglich zur Anwendung und können zu einer Beendigung der Schutzgewährung führen (Art. 19 RL 2011/95). Solange die Beendigung des unionsrechtlichen subsidiären Schutzstatus nicht zur Folge hat, dass die Person in einen Staat ausgewiesen wird, wo sie an Leib und Leben bedroht ist, sind diese Bestimmungen der Richtlinie als völkerrechtskonform einzustufen. Problematisch erscheint aber einmal mehr die Vermischung der Zielsetzungen von Ausschluss- und Ausweisungsbestimmungen. Zudem besteht ein beachtliches Risiko, dass die zuständigen Behörden in den Mitgliedstaaten die notwendigen Differenzierungen zwischen dem absolut geltenden menschenrechtlichen Rückschiebungsschutz und dem einschränkbaren und beendigungsfähigen unionsrechtlichen subsidiären Schutz-

23 S. hierzu etwa Astrid Epiney, Zeitschrift für Ausländerrecht 2/2007, S. 64; Constantin Teetzmann, Europarecht 1/2016, S. 101. Zum Umfang der Bindung des Unionsgesetzgebers an die grund- und völkerrechtlichen Vorgaben s. ausführlicher Egbuna-Joss (Fn. 1), S. 118 ff. m.w.H.

24 S. oben III.1. 
status in der Praxis nur schwer nachvollziehen und umsetzen können. Dies gilt umso mehr, als sich die Anspruchsvoraussetzungen für den menschenrechtlichen Rückschiebungsschutz und den unionsrechtlichen subsidiären Schutzstatus weitestgehend entsprechen.

\section{Würdigung}

27 Diese kurze Erörterung des Inhaltes und der Völkerrechtskonformität derjenigen Bestimmungen der Qualifikationsrichtlinie, welche direkt oder indirekt den Schutz der öffentlichen Sicherheit zum Ziel haben ${ }^{25}$, kommt zum Ergebnis, dass sich diese mit wenigen Ausnahmen ${ }^{26}$ völkerrechtskonform auslegen lassen und sich daher im Rahmen des völkerrechtlich Zulässigen bewegen. Deutlich wird aber, dass die Qualifikationsrichtlinie teilweise völkerrechtliche Konzepte vermischt oder zu vermischen droht, und dass die diskutierten Bestimmungen, welche aus Gründen der nationalen Sicherheit oder öffentlichen Ordnung Einschränkungen der Rechte von Flüchtlingen und subsidiär Schutzberechtigen vorsehen, den Mitgliedstaaten einen erheblichen Beurteilungs- und Ermessenspielraum einräumen.

Die analysierten Bestimmungen der Qualifikationsrichtlinie sehen als Antwort auf das für sicherheitsgefährdend befundene Verhalten vor, dass eine Person entweder von der Schutzgewährung ausgeschlossen (Art. 12 Abs. 2 und Art. 17 Abs. 1 RL 2011/95), ihre Reisefreiheit innerhalb und ausserhalb der EU eingeschränkt (Art. 24 und 25 RL 2011/95) oder ihre

25 S. eine ausführlichere Zusammenfassung der Ergebnisse bei Egbuna-Joss (Fn. 1), §15, Rn. 20 ff.

26 S. Egbuna-Joss (Fn. 1), §15, Rn. 49-52.
Schutzgewährung bei einer tatsächlichen und aktuellen Gefahr sogar beendet werden kann (Art. 14 und Art. 19 RL 2011/95). Von der Richtlinie weiterhin nicht geregelt ist der Status von Personen, die von diesen Bestimmungen erfasst werden. Eindeutig geklärt scheint lediglich, dass auch eine solche Person nicht in ihr Herkunftsland aus- oder zurückgewiesen werden darf, wenn sie dort an Leib und Leben bedroht wäre.

29 Auf diesem menschenrechtlichen Bleiberecht besteht der Europäische Gerichtshof für Menschenrechte gemäss ständiger Rechtsprechung gerade auch bei mutmasslichen Terroristen und weiteren Personen, von denen eine Gefahr für die Sicherheit des Aufenthaltsstaates aus geht ${ }^{27}$. Mehrere Staaten haben sich in neueren Verfahren vor dem Gerichtshof immer wieder gegen diese absolut geltende Vorgabe gewehrt und vorgebracht, dass die absolute Geltung des menschenrechtlichen Refoulement-Verbotes den aktuellen Bedrohungen durch den internationalen Terrorismus nicht angemessen Rechnung trage ${ }^{28}$. Bisher hat der EGMR solche Vorbringen stets zurückgewiesen, und es gibt keine Anzeichen dafür, dass er seine Rechtsprechung in naher Zukunft ändern wird. Aber selbst bei einer Änderung der Rechtsprechung bestehen angesichts der zunehmend globalen Vernetzung und des internationalen Terrorismus ernsthafte Zweifel, ob die Aus- und Zurückweisung von besonders gefährlichen Personen tatsächlich ein geeignetes Mittel wäre, um die innere

27 S. den Überblick bei Egbuna-Joss (Fn. 1), S. 55 ff.

28 Urteil des EGMR 25425/05 vom 27. Mai 2008 (Ramzy gegen Niederlande), Rn. 130; Urteil des EGMR 4900/06 vom 27. Mai 2008 (A. gegen Niederlande), Rn. 130; vgl. Egbuna-Joss (Fn. 1), S. $57 \mathrm{f}$. 
und äussere Sicherheit der Mitgliedstaaten und der Union effektiv zu schützen.

3o Abschliessend bleibt daher festzuhalten, dass die Qualifikationsrichtlinie 2011/95 zwar Bestimmungen enthält, die es den Mitgliedstaaten erlauben, ihre öffentliche Sicherheit und Ordnung auch im Rahmen von Entscheidungen über die internationale Schutzgewährung zu berücksichtigen und grundsätzlich keine zusätzliche Verschärfung der Asylgesetzgebung angezeigt ist. Gleichzeitig wird aber auch deutlich, dass selbst die schärfsten Sanktionen des Asylrechts - die Verweigerung der Schutzgewährung bei Ausschlussgründen, die Beschränkung der Statusrechte und die Beendigung der Schutzgewährung - alleine genommen nicht die geeigneten Mittel sind, um den internationalen Terrorismus effektiv zu bekämpfen. Dies muss weiterhin die primäre Aufgabe der Sicherheitsdienste, der Polizei- und Strafverfolgungsbehörden bleiben. Das Asylrecht kann hier nur eine untergeordnete und unterstützende Rolle spielen. 